\title{
Differential speckle interferometry: in-depth analysis of the solar photosphere ${ }^{\star}$
}

\author{
C. Grec $^{1}$, C. Aime ${ }^{1}$, M. Faurobert ${ }^{1}$, G. Ricort ${ }^{1}$, and F. Paletou ${ }^{2}$ \\ 1 Université de Nice Sophia Antipolis, Laboratoire Universitaire d'Astrophysique de Nice (UMR 6525), Parc Valrose, \\ 06108 Nice Cedex 2, France \\ e-mail: catherine.grec@unice.fr \\ ${ }^{2}$ Université Paul Sabatier, Toulouse 3, Observatoire Midi-Pyrénées, Laboratoire d'Astrophysique (CNRS/UMR 5572), \\ 14 avenue Édouard Belin, 31400 Toulouse, France
}

Received 9 June 2006 / Accepted 20 September 2006

\section{ABSTRACT}

\begin{abstract}
Aims. We present the results of an experiment performed at the solar telescope THEMIS in 2002 to measure the depth over which the solar granulation extends in the photosphere.

Methods. Observations made in the $523.3 \mathrm{~nm}$ and $557.6 \mathrm{~nm}$ photospheric non-magnetic iron lines were correlated with images in the continuum using spectrograms. The difference in depth between the different levels in the photosphere is projected into a difference of position along the slit of the spectrograph, using a perspective effect similar to the well-known Wilson effect for sunspots. This requires measuring displacements, ones much smaller than the telescope resolution. This is made possible by using a differential speckle interferometric technique, cross-correlating images taken in the continuum and the line. The method is not adapted to following displacements of structures in the core of strong lines, due to their difference in shapes with the structures observed in the continuum. In this case, a sequential cross-spectrum method is developed to cross-correlate images taken at close wavelengths.

Results. The raw results are surprising: displacements measured in the blue and the red wings of a line have opposite signs! North and South observations, however, clearly show the expected behavior attributed to a perspective effect. After a description of the observations, we give a first interpretation of the results. The main part of the observed displacement comes from the effect of unresolved Doppler shifts produced by horizontal velocities in the solar photosphere. The perspective effect we seek appears as a second-order term; we find that its amplitude is 2 or 3 times larger than predicted by theoretical 1D models. In the core of strong lines we detect a contrast inversion that also shows up in the cross-correlation function as an anti-correlation peak at line center. Conclusions. This first use of the differential speckle interferometry technique on the Sun is quite promising for 3D studies at high spatial resolution. Further observations with very good image quality are needed to take advantage of this new technique.
\end{abstract}

Key words. line: formation - Sun: granulation - techniques: high angular resolution - Sun: atmosphere

\section{Introduction}

The well-known solar granulation phenomenon is due to the transition between convective and radiative regimes that exists near the base of the photosphere. Hot gas coming from the convective zone is suddenly cooled by radiation. The photospheric visible result is an evolving cell pattern. The bright hot gas rises in granules, extends horizontally, and sinks with very turbulent behavior in the dark intergranular lanes after it has cooled. In late-type stars like the Sun, the convective zone extends to the visible surface layers and influences the emergent spectrum, thereby shifting the spectral lines and modifying their shapes and symmetries.

For more than a decade, thanks to technological advances in computers, 3D radiative hydrodynamical simulations of convection have been developed. Nowadays, one can simulate the formation of different solar spectral lines with a 3D model atmosphere and then compare the resulting line profiles, shifts, and asymmetries with the observed ones. A very good agreement is obtained between simulation and line-profiles observed at

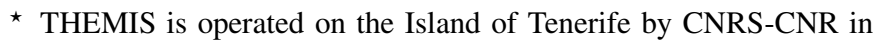
the Spanish Observatorio del Teide of the Instituto de Astrofísica de Canarias. moderate spatial resolution (Asplund et al. 2000); those 3D models' atmospheres should be more precisely constrained by high resolution observations showing the granules behavior, from the deepest photospheric layers to the upper photosphere.

In the past, spectroscopic methods have been extensively used to study the vertical structure of the photosphere. Recently, Eibe et al. (2001) explored the diagnostic properties of the $\mathrm{Na}$ ID resonance line by calculating the response functions of the line profile to temperature and velocity perturbations in the atmosphere. The response functions are quite broad because of non-LTE effects in the Na ID line formation. The depth structure of the photosphere is not well-resolved by this method.

Here, we implement for the first time in solar physics a differential speckle interferometry (DSI) high-resolution technique. This allows us to very precisely determine the line formation depth and the depth-range on which photospheric structures remain similar to themselves: this is one way of having $3 \mathrm{D}$ images of the photosphere. DSI belongs to speckle interferometry (SI) techniques but is quite different in the result it gives.

SI techniques (Labeyrie 1970) applied to the solar photosphere (Harvey \& Breckinridge 1973) permit the spatial power spectrum of the solar granulation to be obtained. The technique is not seeing-limited; if the signal-to-noise ratio is good 
enough, it should give information for spatial frequencies up to the diffraction limit of the telescope. The difficulty with this method stems from the fact that, to get useful results, the effect of the atmospheric turbulence must be estimated and corrected (Ricort \& Aime 1979; Aime 1974). Moreover, since SI does not provide images, a morphological interpretation of the power spectrum must be made to provide astrophysical results (Aime 1978).

Speckle imaging techniques (Knox \& Thompson 1974; Weigelt 1977) allow both high angular resolution and direct images to be obtained. Their application to the solar photosphere was developed by Von der Lühe (Von der Lühe \& Pehlemann 1988) and by the Göttingen group (Wilken et al. 1997; Krieg et al. 2000) in several studies.

The DSI technique was first proposed by Beckers \& Hege (1982). It allows us to measure shifts between structures when the shifts are much smaller than the telescope Airy disk. This technique is limited neither by the seeing nor by the diffraction. In the presence of atmospheric turbulence, the measurement is possible with the basic assumptions of SI. The main interest of DSI is that the information is held by the phase of the crossspectrum and that no calibration of the modulation transfer function is needed. The DSI method was developed many years ago at the University of Nice (Aime et al. 1984, 1986) for stellar applications.

This paper is organized as follows. The second section describes the method and its general performance. The third section presents the observations and data processing. Results are given in the fourth section and interpreted with the help of modeling residual velocity fields in Sect. 5. Finally, conclusions are given in Sect. 6.

\section{Differential speckle interferometry on the Sun}

Our experiment is based on the fact that two overlapping structures in the photosphere, observed outside the disc center, will appear horizontally displaced when one is compared to the other. The displacement $\varepsilon$ is proportional to the difference of depth $\Delta h$ between the structures and to the projected distance $r$ from the solar center,

$\varepsilon=\Delta h \times \frac{r}{R_{\odot}}=\Delta h \times \sin \theta$,

where $\theta$ is the usual angle between the position and the viewsight. Expected shifts are very small. For example, taking $\sin \theta=0.5$ and observing in a weak line $(\Delta h=50 \mathrm{~km})$, the shift should be of $25 \mathrm{~km}$ or $0.034^{\prime \prime}$, which is far smaller than the telescope's theoretical resolution. However, as the measure of a shift between two images is a differential measurement, it is not limited by diffraction effects.

A very schematic illustration of the experiment showing the perspective effect for a cylindrical granule seen at two different levels is represented in Fig. 1. In practice, we observed regions of the solar surface of $70 \times 70$ arcsec centered on the position $r=0.5$ and $0.75 R_{\odot}$ in the North and South hemispheres. Orienting the $x$-axis in the South-North direction, we expect $\varepsilon$ to be positive for observations in the North hemisphere and negative for observations in the South hemisphere.

We describe below how shifts can be measured using a statistical analysis.

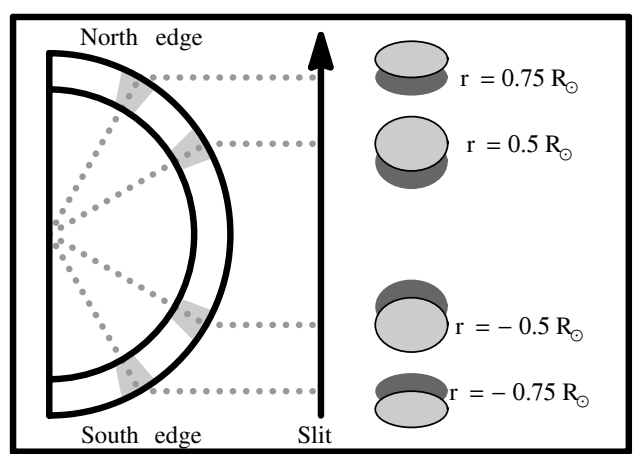

Fig. 1. Perspective effect for different positions on the solar disc: schematic representation of the displacements of a photospheric structure. Here the structure is assumed to be a cylinder. The dark-gray section of the structure corresponds to the observation in the continuum; the light-gray one corresponds to the observation in the line. By projection, the closer to the solar edge that the observation is done, the larger the shifts between the structures. Note that shifts have opposite directions for North and South observations.

\subsection{Two dimensional considerations}

Let us denote the photospheric brightness fluctuation of the solar surface by $B_{\lambda}(x, y)$. An observation $B_{\mathrm{W}}(x, y)$ made in the wing of a line corresponds to a higher formation level in the photosphere than the image $B_{\mathrm{C}}(x, y)$ taken in the continuum of the spectrum. Due to a perspective effect outside the disk center $B_{\mathrm{W}}(x, y)$ will appear shifted towards the limb in comparison to $B_{\mathrm{C}}(x, y)$. If we make the assumption that the structures are similar, we can write

$B_{\mathrm{W}}(x, y) \sim B_{\mathrm{C}}(x-\varepsilon, y)$,

where the $x$-axis is perpendicular to the limb.

In the telescope's focal plane we observe the image $I_{\lambda}(x, y)$, which is the convolution of the geometrical image of $B_{\lambda}(x, y)$ with the telescope-atmosphere point spread function $S(x, y)$. We have

$I_{\lambda}(x, y)=B_{\lambda}(x, y) * S(x, y)$,

where the symbol $*$ stands for convolution. Within the $5 \AA$ spectral window of our analysis, the function $S(x, y)$ can be considered as wavelength independent. Thanks to the linear property of the convolution operation, the resulting image is shifted by the same value $\varepsilon$ as the object, and we can write

$I_{\mathrm{W}}(x, y) \sim I_{\mathrm{C}}(x-\varepsilon, y)$.

Defining the Fourier transform as

$\widehat{f}(u, v)=\iint f(x, y) \exp 2 \mathrm{i} \pi(u x+v y) \mathrm{d} x \mathrm{~d} y$,

relation (4) becomes in Fourier space

$\widehat{I}_{\mathrm{W}}(u, v) \sim \widehat{I}_{\mathrm{C}}(u, v) \exp (2 \mathrm{i} \pi u \varepsilon)$.

The displacement introduces a deterministic linear phase term $2 \pi \varepsilon u$, proportional to the angular frequency $u$. For example, a $25 \mathrm{~km}$ shift will produce a phase shift of $\varphi=2 \pi * 25 / 727 \mathrm{rad} \simeq$ $0.22 \mathrm{rad}$ at $u=1 \mathrm{c} / \mathrm{s}$ (cycle per arcsec).

The effects of a 2D detector could be taken further into account. One of them is the sampling of the spectrogram that might produce aliasing in the Fourier space. This effect is probably negligible since the best images have shown a signal up to about $75 \%$ of the frequency cutoff. In any case these effects are 
limited by the size of each elementary pixel of the CCD that, by smoothing the resulting image of Eq. (3), will act as a low pass filter for the spectrum, with no consequence for the measurement of $\varepsilon$ we seek.

\subsection{One-dimensional considerations}

Because we use spectrograms, we only have access to the $1 \mathrm{D}$ brightness intensity along the slit, which is aligned with the $x$-axis. We thus obtain the value of $I_{\lambda}(x, y)$ for a fixed $y=y_{0}$; we can take $y_{0}=0$ with no loss in generality.

Although we recorded spectrograms for different values of $y$ in a slow scanning mode (within a zone of 70 arcsec), we made no attempt to recover the 2D spatial information in our experiment. It would indeed be difficult to recover that information from records obtained at different times, and the displacement $\varepsilon$ we seek can be perfectly extracted from the 1D analysis. We recorded many spectrograms at different $y$ positions to improve the statistics of the solar granulation power spectrum. This quantity plays an important role in our analysis, as we shall see later (Eq. (11)).

Denoting $I_{\lambda}(x)$ as the $1 \mathrm{D}$ cuts $I_{\lambda}(x, 0)$ of the images for simplicity, we can write

$I_{\mathrm{W}}(x) \sim I_{\mathrm{C}}(x-\varepsilon)$

and

$\widehat{I}_{\mathrm{W}}(u) \sim \widehat{I}_{\mathrm{C}}(u) \exp (2 \mathrm{i} \pi u \varepsilon)$.

It may be interesting to note that $\widehat{I}_{\lambda}(u)$ derives from $\widehat{I}_{\lambda}(u, v)$ by the relation

$\widehat{I}_{\lambda}(u)=\int \widehat{I}_{\lambda}(u, v) \mathrm{d} v$.

This can be easily demonstrated by writing $I_{\lambda}(x, 0)$ as the inverse Fourier transform of $\widehat{I}_{\lambda}(u, v)$ for $y=0$.

\subsection{Cross-spectrum technique}

Shifts can be derived from the phase of the cross-spectrum. We use a series of spectrograms to estimate the cross-spectrum $\widehat{Q}_{\mathrm{CW}}$ between $I_{\mathrm{C}}(x)$ and $I_{\mathrm{W}}(x)$ :

$$
\begin{aligned}
\widehat{Q}_{\mathrm{CW}}(u) & =\left\langle\widehat{I}_{\mathrm{C}}(u) \widehat{I}_{\mathrm{W}}^{*}(u)\right\rangle \\
& \sim\left\langle\left.\widehat{I}_{\mathrm{C}}(u)\right|^{2}\right\rangle \mathrm{e}^{-2 \mathrm{i} \pi \varepsilon u},
\end{aligned}
$$

where $\widehat{I_{\mathrm{W}}^{*}}(u)$ represents the conjugate Fourier transform of $I_{\mathrm{W}}(x)$, and the brackets refer to the ensemble average. With the assumption of similar image shapes in the line and the continuum, the amplitude of the cross-spectrum $\left\langle\left.\widehat{I}_{\mathrm{C}}(u)\right|^{2}\right\rangle$ is identical to the power spectrum of the granulation, attenuated by the instrument/atmosphere modulation transfer function (Aime 1974).

We emphasize that the interest of this cross-spectrum analysis is to produce the complex number $\widehat{Q}_{\mathrm{CW}}(u)$, whose phase gives exactly the required information. The alert reader will have noted that this information was not available in $\widehat{I}_{\mathrm{W}}(u, v)$ or in $\widehat{I}_{\mathrm{W}}(u)$ of Eqs. (6) and (8) that are contaminated by the phases of $\widehat{I}_{\mathrm{C}}(u, v)$ and $\widehat{I}_{\mathrm{C}}(u)$. Limits for the measurement have two origins. The first one comes from the diffraction and the effects of the atmospheric turbulence. But we also need to have good statistics on the granulation itself. This implies there should be no sharp structures in the observed power spectrum. Relation (9) shows that $\left\langle\left.\widehat{I}_{\mathrm{C}}(u)\right|^{2}\right\rangle$ is the integrated value of $\left\langle\left|\widehat{I}_{C}(u, v)\right|^{2}\right\rangle$. This latter quantity can be written as the following product

$\left\langle\left|\widehat{I}_{\mathrm{C}}(u, v)\right|^{2}\right\rangle=\left\langle\left|\widehat{B}_{\mathrm{C}}(u, v)\right|^{2}\right\rangle\left\langle\left|\widehat{S}_{\mathrm{C}}(u, v)\right|^{2}\right\rangle$,

where $\left\langle\left|\widehat{B}_{C}(u, v)\right|^{2}\right\rangle$ is the solar granulation power spectrum and $\left\langle\left|\widehat{S}_{\mathrm{C}}(u, v)\right|^{2}\right\rangle$ is the lens-atmosphere energy transfer function. To get the best measurement for the linear phase term $2 \mathrm{i} \pi u$, these quantities should be as smooth as possible, which means that we need the best possible ensemble average for the atmospheric turbulence, but also for the solar granulation.

The similarity of the images in the line and in the continuum is essential for these measurements. This condition is no longer verified near the center of strong absorption lines formed over large depths in the photosphere. In the section "First Results", we present and further discuss an example of the phase showing $\pi$ phase rupture, for which the interpretation in terms of shifts is not straightforward. In such a situation, the depth variation of the granular structures is investigated better by calculating crossspectra between images taken at two nearby wavelengths, for example at $\lambda$ and $\lambda+\Delta \lambda$, where $\lambda$ is allowed to scan the entire region of interest in the spectrum. This permits us to compare more similar structures through the line, due to the closer heights of formation. In that case, Eq. (10) becomes:

$$
\begin{aligned}
\widehat{Q}_{\lambda \Delta \lambda}(u) & =\left\langle\widehat{I}_{\lambda}(u) \widehat{I}_{\lambda+\Delta \lambda}^{*}(u)\right\rangle \\
& \sim\left\langle\left.\widehat{I_{\lambda}}(u)\right|^{2}\right\rangle \mathrm{e}^{-2 \mathrm{i} \pi \varepsilon^{\prime} u},
\end{aligned}
$$

where $\left\langle\left|\widehat{I}_{\lambda}(u)\right|^{2}\right\rangle$ is the power spectrum of the photospheric structure observed at the wavelength $\lambda$. The gain in this "sequential cross-spectrum" procedure is a better resemblance between the images, while the drawback is that we have to measure a displacement $\varepsilon^{\prime}$ that is much smaller than $\varepsilon$.

For illustration, we give in Figs. 2 and 3 examples of crossspectra (modulus and phase) allowing to derive $\varepsilon$ and $\varepsilon^{\prime}$. These cross-spectra are ensemble averages calculated using 266 spectrograms. Later in the paper, we describe the detailed procedure we used to obtain these results. Shifts between structures are given by the slope of the cross-spectrum phase. The expected linear behavior is clearly achieved here in the spatial frequency domain between 0.2 and $0.78 \mathrm{c} / \mathrm{s}$.

At high spatial frequencies, the phase falls to 0 instead of showing the expected random variation between $\pm \pi$ due to the noise, as seen in stellar measurements (Aime et al. 1984). We interpret this behavior as uncorrected residues in the flat-fielding of the image. Another spurious effect is the break at very low frequencies; it is interpreted as a smoothing effect of the crossspectrum. Both effects are explained in Appendix A.

\subsection{Cross-correlation technique}

The cross-correlation function is defined by the integral

$K_{\mathrm{CW}}(\tau)=\left\langle\int I_{\mathrm{W}}(x) I_{\mathrm{C}}(x-\tau) \mathrm{d} x\right\rangle$.

This function is generally used to check similarities between structures (Janssen \& Cauzzi 2006), and it can be used to determine the displacements of features (Prieur et al. 2004). In the assumptions of similarities of structures for Eq. (2), $K_{\mathrm{CW}}(\tau)$ shows a maximum for $\tau$ equal to $\varepsilon$, and the shift between structures is therefore given simply by the position of the cross-correlation maximum.

As a result of the Wiener Khinchine theorem, the crosscorrelation is also the Fourier transform of the cross-spectrum, 

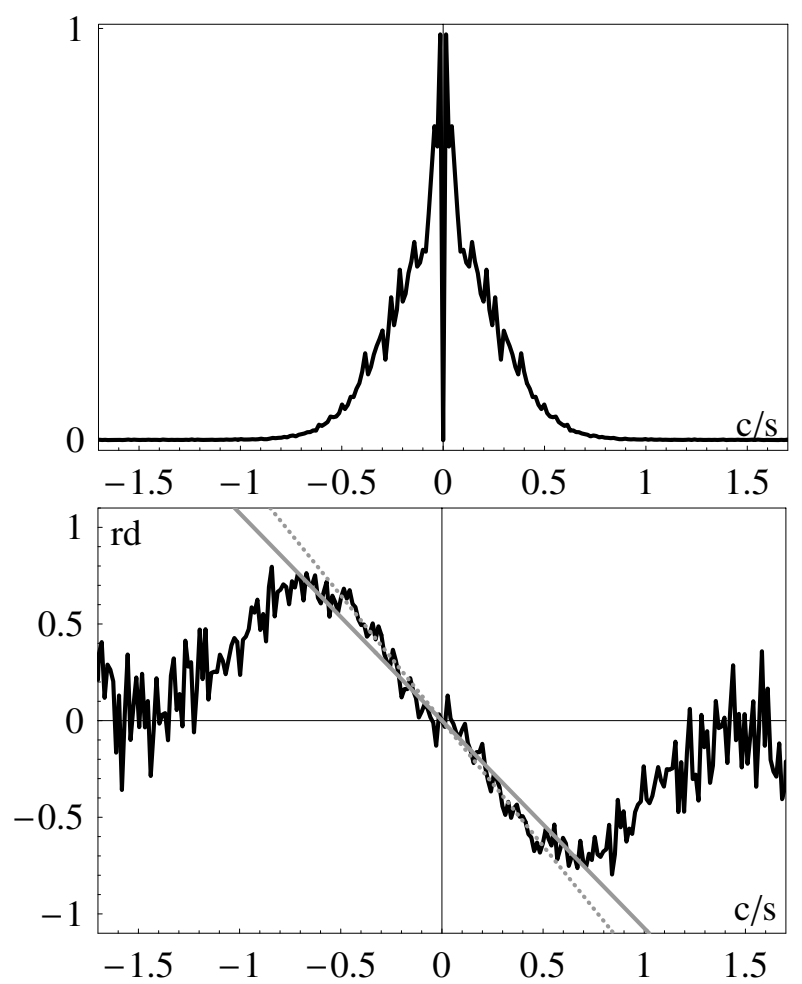

Fig. 2. Example of determination of the displacement $\varepsilon$ between line and continuum structures: phase (bottom) and log-scale modulus (top) of the cross-spectrum, calculated between the continuum and a narrow channel in the blue wing of the $557.6 \mathrm{~nm}$ line (for the $21 \%$ line depression coefficient). The $x$-axis is in $\operatorname{arcsec}{ }^{-1}$. The phase has the expected linear curve in the spatial frequency domain corresponding to a significative power of the cross-spectrum modulus. The phase given here corresponds to a displacement of about $0.2 \mathrm{c} / \mathrm{s}$ (phase of $1.3 \mathrm{rad}$ at $1 \mathrm{c} / \mathrm{s}$ ).

and these two functions contain equivalent information. In practice, it is more convenient to use the cross-spectrum to measure much smaller displacements than the pixel size and the crosscorrelation for large motions.

For our analysis, we used both techniques; we found the cross-correlation useful for analyzing the motion of structures on large scales. The cross-correlation, which is a quantity belonging to the real domain, is also easier to interpret when differences between structures cannot be reduced to a simple shift.

\section{Observations and data processing}

Our data material consisted in high-spectral resolution $x \times \lambda$ spectrograms, obtained on a quiet Sun at the THEMIS telescope (Izaña, Canarias) in September 2002. The direction of the spectrograph slit was set perpendicular to the solar edge and along the North-South axis to avoid Doppler effects resulting from the Sun's rotation. The observations were done for four regions on the solar surface, around the positions $r=0.5 R_{\odot}(\mu=0.86)$ and $r=0.75 R_{\odot}(\mu=0.66)$ in the North and in the South hemispheres.

The solar spectrum was observed in two $5 \AA$ regions around the $523.3 \mathrm{~nm}$ and $557.6 \mathrm{~nm}$ non magnetic iron lines; the wavelength sampling was of $12.5 \mathrm{~m} \AA / \mathrm{px}$. The spectrograph slit was $70^{\prime \prime}$ long, with a pixel sampling of $0.29^{\prime \prime}$, which was unfortunately not satisfactory since it was about four times too large for the theoretical resolution of the 90-cm THEMIS telescope $\left(0.134^{\prime \prime}\right.$ at $\left.589 \mathrm{~nm}\right)$. Its actual resolution was thus reduced to that of a $20 \mathrm{~cm}$ telescope.

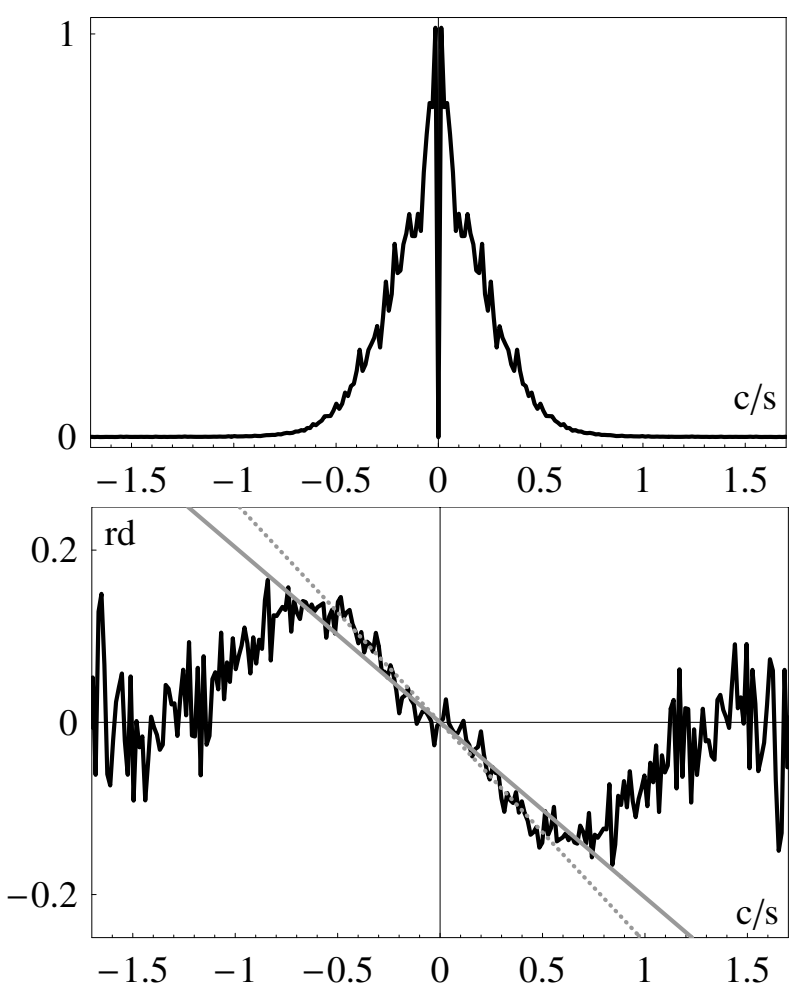

Fig. 3. Example of determination of the displacement $\varepsilon^{\prime}$ between structures observed for close wavelengths on the line: phase (bottom) and log-scale modulus (top) of the cross-spectrum calculated between two close wavelengths near the $557.6 \mathrm{~nm}$ line center (respectively for the $16 \%$ and $21 \%$ line depression coefficient). As expected, the slope of the phase we measure is smaller than in Fig. 2, because we compare close levels in the photosphere.

To improve the statistic, the slit scanned a region of about $70 \times 70$ arcsec, keeping its orientation fixed along the NorthSouth direction. The slit was strictly perpendicular to the local solar limb only for the central position of the scan. However its tilt with respect to the perpendicular remained smaller than $3^{\circ}$, we could thus always assume that the perspective effect induced displacements along the slit direction. During a run, we scanned the $70 \times 70$ arcsec region, recording 200 spectrograms. The Doppler effect due to Sun's rotation was negligible on this region.

The exposure time was $300 \mathrm{~ms}$, which was clearly not short enough to freeze the atmospheric turbulence. Speckle boiling produces a smearing effect that tends to destroy the highest spatial frequencies. Nevertheless, a careful examination of the data allowed us to select 659 spectrograms that still present sufficient energy in the high frequencies to make the measurement possible, thanks to quite a high value for the $r_{0}$ parameter and a relatively low speed turbulence. We selected images with a contrast criterium. A relation between contrast and $r_{0}$ values exists (Ricort \& Aime 1979). The maximal spatial frequency detectable in our images was $1.28 \mathrm{c} / \mathrm{s}$, which corresponds to a spatial scale of 0.78 arcsec. Having too long an exposure time made it more difficult to determine the phase because of signalto-noise ratio problems. Hopefully this does not bias the phase measurement, since the effect of the time integration is linear.

In a given $\lambda$-domain, we can easily detect a misalignment between the CCD and the spectrograph apparatus by using the cross-spectrum technique itself (Eq. (10)). It produces a deterministic displacement that biases the measurement of the 


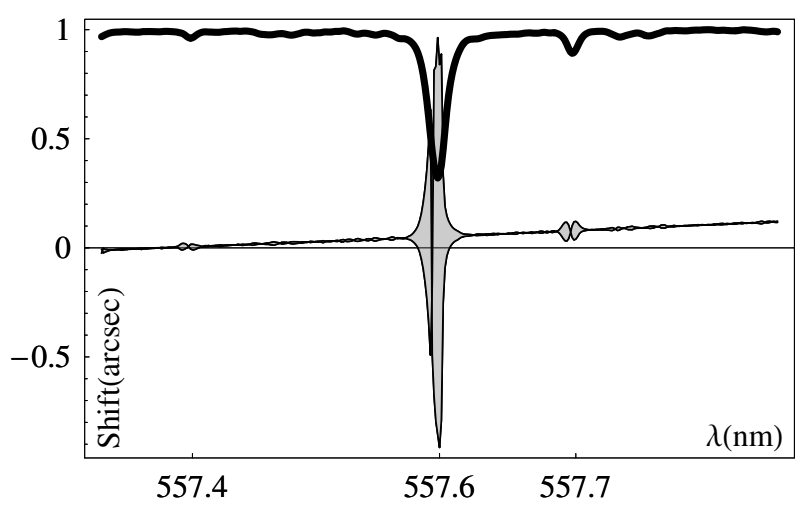

Fig. 4. Dark line: line profiles on the $557.6 \mathrm{~nm}$ domain. Gray lines (filled-in): biased shifts measured at the North and South positions, at $\mu=0.5$, as a function of $\lambda$.

cross-spectrum phase. This method allowed us to detect a misalignment of the CCD used for the $557.6 \mathrm{~nm}$ domain ( 0.5 pixel between the two edges of the $\mathrm{CCD}$, which corresponds to a small $3.4^{\prime}$ misalignment) and to determine its effect on the whole $\lambda$-domain (see Fig. 4). This bias is corrected a posteriori by fitting the linear trend. In the following, we present the results for the shifts after this correction.

\subsection{Pre-processing}

A first step in the processing was the Doppler-shift compensation in the spectral lines between granular and intergranular regions (see Fig. 5). Since we want to relate an image of the granulation at a given wavelength to a given photospheric layer (i.e. to a given line depression coefficient), we have to re-center the line on the same reference wavelength for each position on the slit. Let us stress here that this Doppler shift correction is a crucial step; see Janssen \& Cauzzi (2006), for an illustration of the problems arising when the correction is not implemented.

We determined the positions of the line center along the slit by calculating its minimum using a polynomial fitting. We empirically chose a 6-degree polynomial as a compromise between the noise sensitivity and the similarity to the data. Then, for each $x$ position on the spectrogram, the entire CCD-row is translated to a referenced wavelength using a Fourier transform. We proceeded as follows. Denoting $\Delta \lambda_{i}$ as the Doppler shift we have to correct, we multiplied the Fourier transform of the row with the phasor $\exp \left(2 \mathrm{i} \pi u \Delta \lambda_{i} / \Delta \lambda_{\text {pixel }}\right)$ and then obtained the translated line profile from the inverse Fourier transform. Note that, with this method, we applied the same Doppler velocity correction for each line level. For weak lines, we can consider that granular velocities do not vary much with depth, so that this correction is sufficient. Strong lines would probably benefit from a more sophisticated treatment than was used here (P. Mein, private communication).

One could also determine the position of the center of gravity for the line, which would give more weight to the whole profile. However the minimum of the line profile, i.e. the maximum of line absorption, corresponds to the uppermost layer of the atmosphere. It is a well-defined quantity, and its position is easily determined by the fitting procedure that we described above.

\subsection{Processing: phase-term determination}

Optimized procedures have been developed to measure differential shifts from the phase of the cross-spectrum in the case of
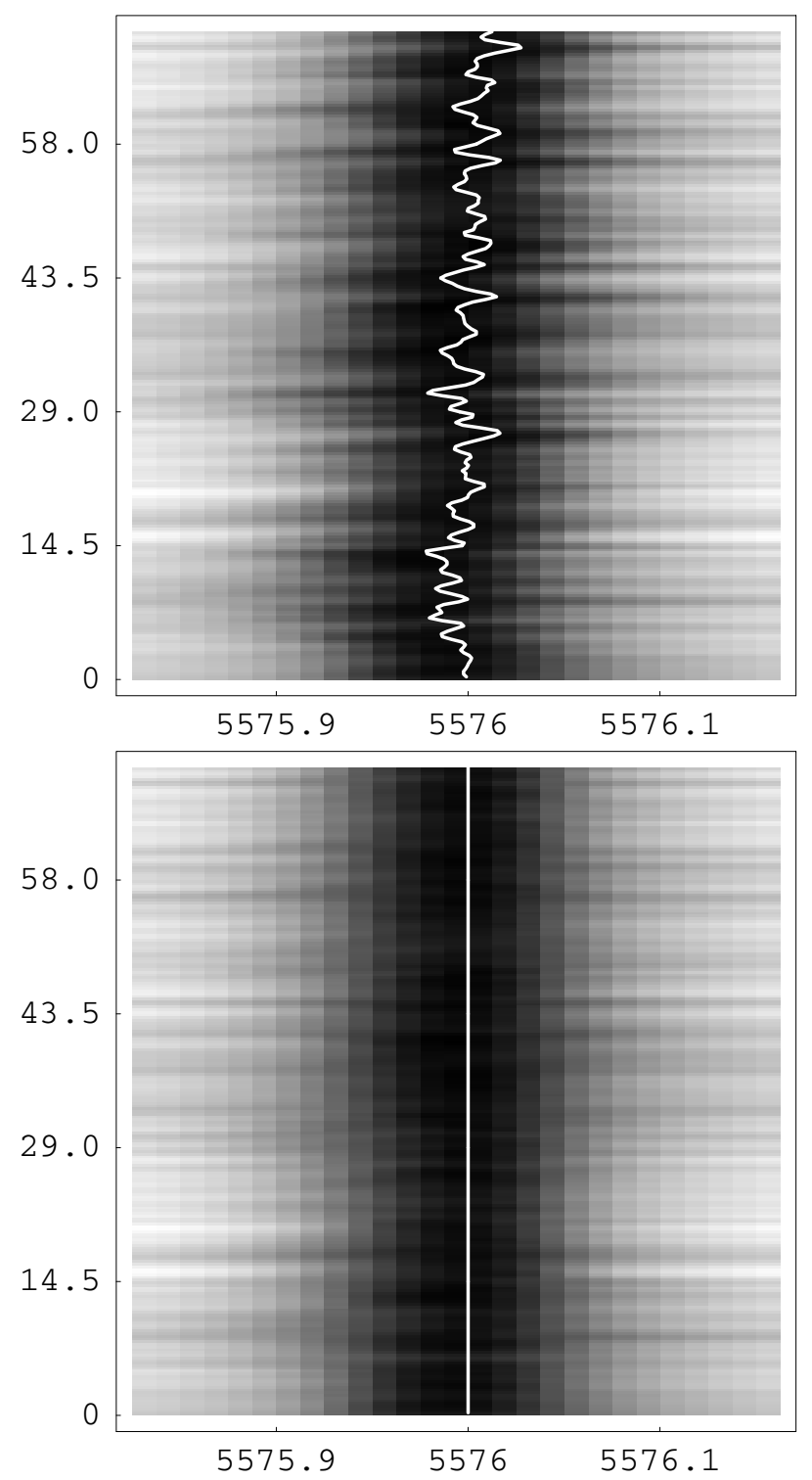

Fig. 5. An example of a $x \times \lambda$ spectrogram recorded around the $557.6 \mathrm{~nm}$ iron line. Along the slit (ordinates), one pixel corresponds to 0.29 arcsec. The wavelength sampling is of $12.5 \mathrm{~m} \AA / \mathrm{px}$ (abscissa). Top: raw image showing the granular and intergranular Doppler effects. Bottom: the same spectrogram, corrected for these velocity fields.

stellar DSI (Petrov et al. 1986). It assumes that observations are limited by photon-noise. This is not the case for our solar observation; and since a complete signal analysis would be difficult to undertake, we used two similar simplified procedures to estimate the slope of the phase. It consists in determining the slope of the phase with a linear fitting that takes into account either a limited range of frequencies (those for which the signal exceeds the noise) or the whole range weighted by the amplitude of the cross-spectrum. In this case, the highest signal frequencies weakly contribute to the slope determination, when noise begins dominating signal. The first technique seems to be more robust, particularly when we determine the slope of the phase obtained at a wavelength near the line center. 


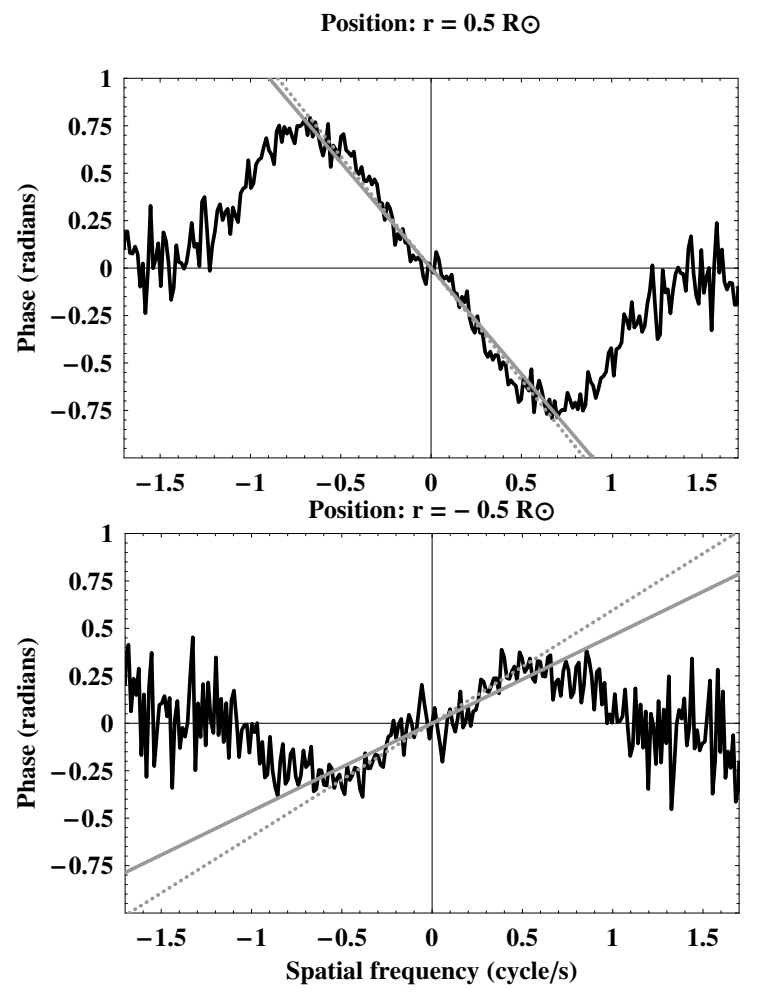

Fig. 6. Expected effect: opposite phases for opposite positions on the Sun disc $(\sin \theta= \pm 0.5)$. Cross-spectra have been calculated on the $557.6 \mathrm{~nm}$ line, between the continuum and a wavelength in the blue wing (line depression coefficient equals to 0.49). The slope of the phase is determined using two linear regression methods (gray and dotted straight lines).

\section{First results}

The first results reveal expected and unexpected phase behaviors. The North-South opposition is recovered, but at one given position we find opposite phase shifts in the red and in the blue wings; in the core of strong lines we detect a phase jump, which is related to a contrast inversion.

\subsection{Expected behavior: north-south opposition}

As illustrated by Fig. 1, we expected to obtain displacements with opposite directions for the observations made at the North and South latitudes. It is indeed what we observe in Fig. 6, which shows an example of the phase of the continuum/line wing crossspectrum obtained for two opposite positions on the sun disk at $r= \pm 0.5 R_{\odot}$ on the Fe I line at $557.6 \mathrm{~nm}$. All the results are similar: the sign of the phase systematically takes opposite values for symmetric North and South positions, as expected from the perspective effect.

\subsection{Unexpected behavior: blue-red opposition of the shifts in the lines}

A completely unexpected effect was found: whatever the spectral line or the position on the Sun disk, the phases have opposite signs in the blue and red wings of the line! This is shown by an example in Fig. 7. Then in Figs. 8 and 9, we show the shifts in arcseconds that we derive from the phases measured for the positions $r= \pm 0.5 R_{\odot}$ in the $557.6 \mathrm{~nm}$ and $523.3 \mathrm{~nm}$ spectral
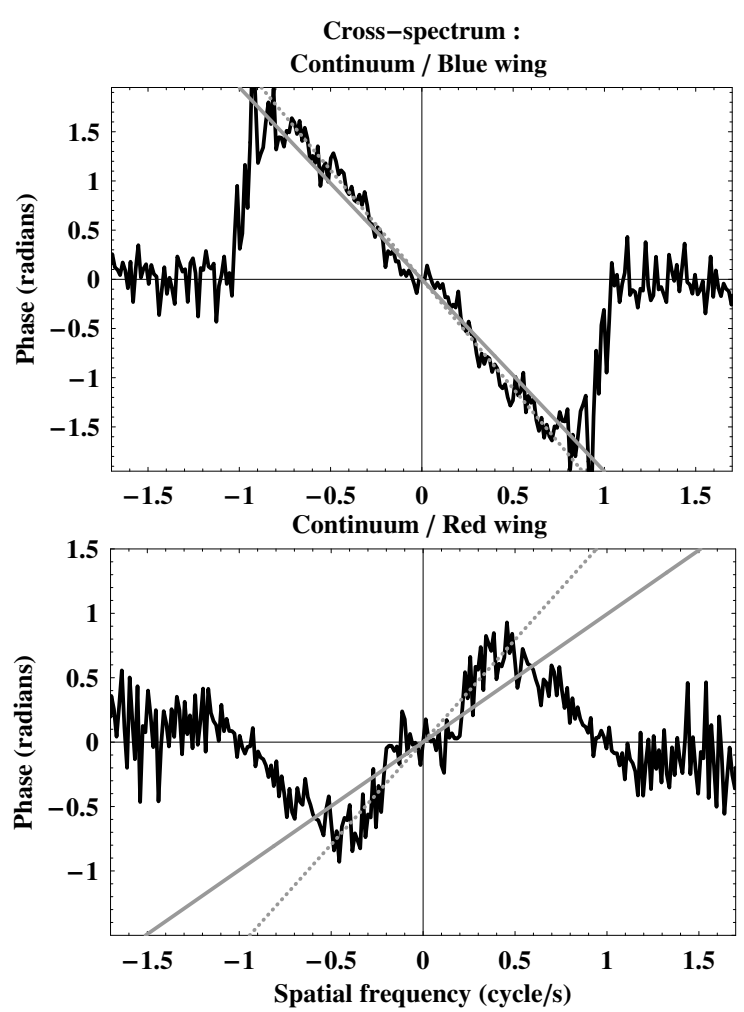

Fig. 7. Unexpected effect: opposite phases in the two wings of the $557.6 \mathrm{~nm}$ line. Top figure (resp. bottom figure): cross-spectrum have been calculated for the same position $r=0.5 R_{\odot}$ between the continuum and a wavelength in the blue (resp. red) wing for a line depression coefficient of 0.33 (resp. 0.25).
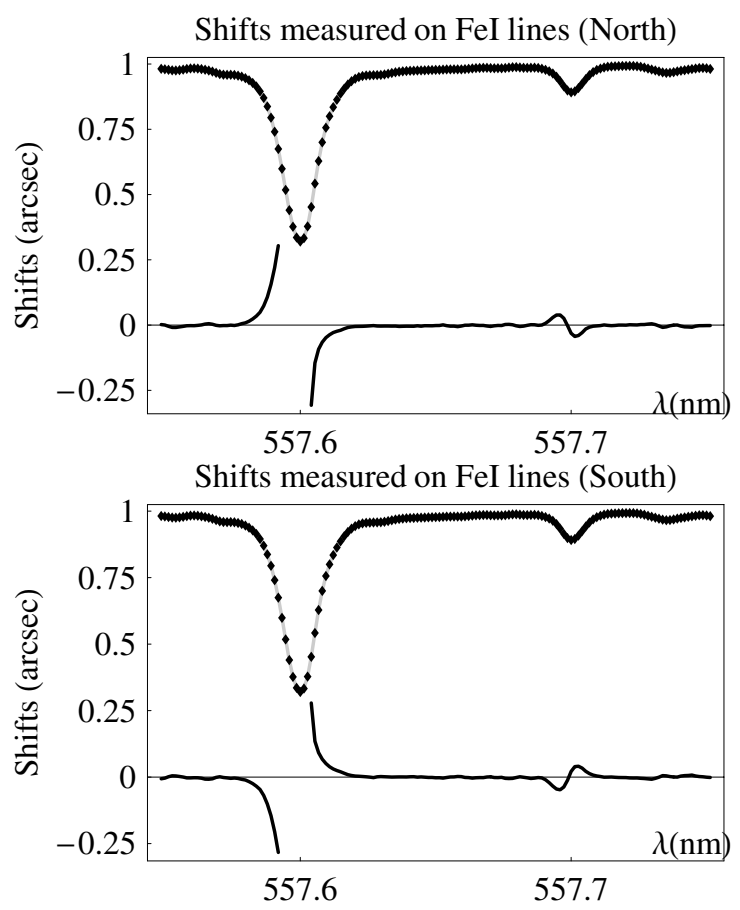

Fig. 8. Shifts measured in the whole CCD $\lambda$-domain around $557.6 \mathrm{~nm}$, for $r=0.5 R_{\odot}(t o p)$ and $r=-0.5 R_{\odot}$ (bottom). Measures are completely consistent: notice the opposite signs of the shifts for North and South positions. The line profile is shown in arbitrary units. In weak lines, the slope of the cross-spectrum phase is determined quite easily. This is not the case near the core of strong lines (values not drawn there). See explanation in the body of the paper. 


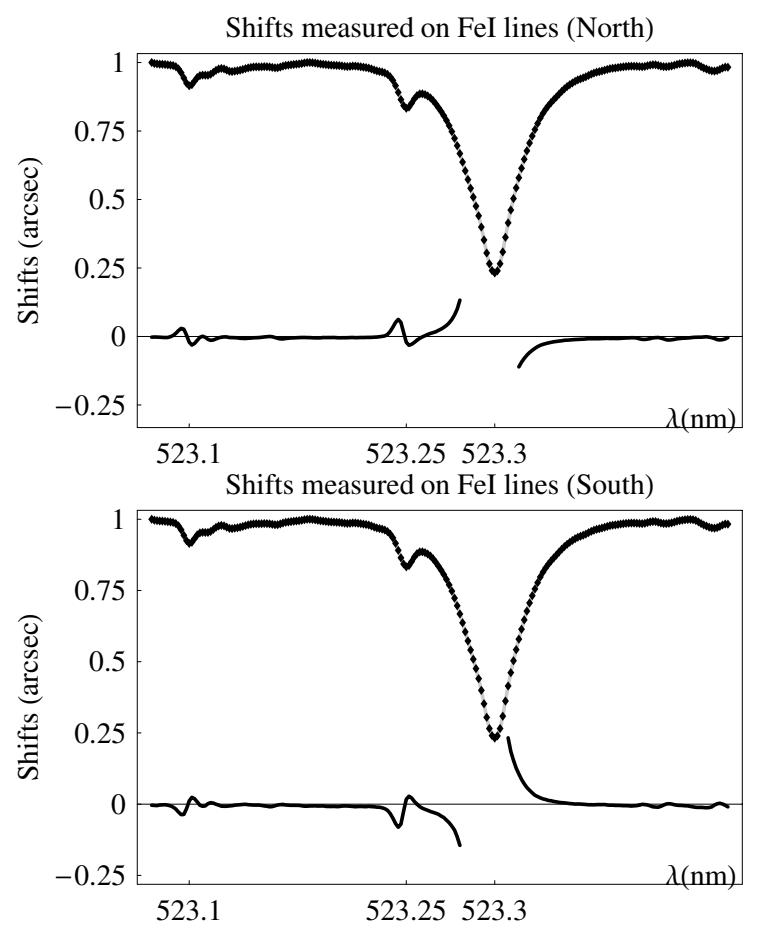

Fig. 9. Shifts measured in the whole $\operatorname{CCD} \lambda$-domain around $523.3 \mathrm{~nm}$, for $r=0.5 R_{\odot}$ (top) and $r=-0.5 R_{\odot}$ (bottom). The meaning of the curves is the same as in Fig. 8.

domains, respectively. We give a physical explanation of this effect in the next section.

\subsection{Phase jump in the core of strong lines and anti-correlation of high angular structures}

Figure 10 illustrates the problems that occur in the core of strong lines. It shows the phase determined between the continuum and a wavelength in the core of the $557.6 \mathrm{~nm}$ line (for a line depression coefficient $\delta(\lambda)=0.39$ ). Note that we have plotted the phase $\pm 2 \pi$ on the same graph, which allows a visual unwrapping of the phase. We see that, for spatial frequencies around $0.4 \mathrm{cycle} / \mathrm{s}$, the phase seems to vary randomly between $\pm \pi$, which coincides with a strong decrease in the cross-spectrum modulus. Such behavior denotes that the phase actually varies by a small quantity around $+\pi($ or $-\pi)$. As the phase calculation always gives results in the interval between $-\pi$ and $+\pi$ (principal value of the phase), it is artificially ascribed a value close to $-\pi$ when it gets larger than $\pi$. This is a well-known problem; it is necessary to "unwrap" the phase in such situations in order to get its correct value. The "unwrapping" consists in adding $2 \pi$ or $-2 \pi$ to the so-called principal value of the phase.

In Fig. 10 we show that the unwrapping of the phase in the spatial frequency range between 0.3 and 0.5 cycle/s (respectively -0.3 and -0.5 cycle/s) amounts to replacing the phase values around $-\pi$ (respectively $+\pi$ ) by values around $+\pi$ (respectively $-\pi)$. We then observe a stepwise monotonous behavior of the phase, with a jump of $+\pi$ (respectively $-\pi$ ) at a spatial frequency around $0.4 \mathrm{cycle} / \mathrm{s}$ (respectively $-0.4 \mathrm{cycle} / \mathrm{s}$ ). This jump of the phase can be due to a contrast inversion of the fine-scale granular structures between the deep layers of the photosphere and the upper layers where the line core is formed. This phenomenon was recently reported by Janssen \& Cauzzi (2006) from high angular resolution images obtained at different wavelengths along the profile of the Fe I line at $709.04 \mathrm{~nm}$. Such a reversal is present in

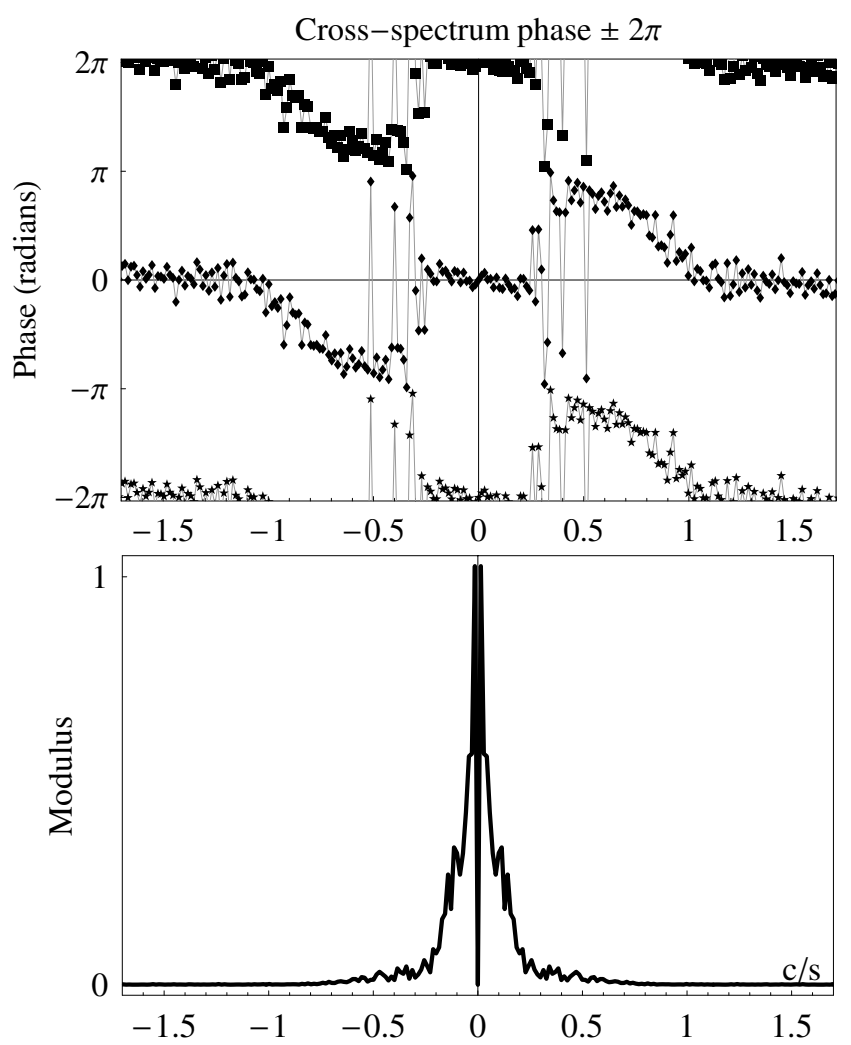

Fig. 10. Phase determination with the usual cross-spectrum technique, for the position $\left(r=0.5 R_{\odot}\right.$ ) on the $557.6 \mathrm{~nm}$ strong line (minimal value of the line depth coefficient: 0.33 ). The phase is calculated between the continuum and a wavelength corresponding to a 0.39 line depression coefficient, and plotted modulo $2 \pi$. See explanation in the body of the paper.

numerical simulations of the photospheric convection (Nordlund 1985). It is the consequence of the granules cooling due to their adiabatic expansion.

In order to check this interpretation, we calculated the crosscorrelations between the 1D intensity variations existing in the continuum and at different wavelengths along the strong $\mathrm{Fe}$ I $557.6 \mathrm{~nm}$ line (we simply calculate the Fourier transform of the cross-spectrum). The result is shown in Fig. 11 for two symmetrical positions on the solar disk. We note that we obtain symmetric figures of cross-correlation for opposite positions on the Sun. The evolution of the cross-correlation through the line is quite complicated. Close to the continuum, we see that the structures are simply displaced in opposite directions on the two wings of the line. But near the line core, an anti-correlation peak is detected. This is a signature of a contrast inversion between the images of the lower and upper photospheres. Let us point out that, from our cross-spectrum analysis, we find that contrast inversion does not affect the larger scales of the photospheric structure but only the scales smaller than 2.5 arcsec, which are typical granular scales.

A consequence of the phase jump in the core of strong lines is that the measurement of the shift between structures seen in the continuum and in the line core by a linear fitting of the phase of the cross-spectrum is no longer valid. The use of the sequential cross-spectrum presented in Sect. 2 is better-suited to these situations because it allows the shifts of the structures to be followed step by step from one layer to the other in the photosphere.

In the following section we present a simple model that accounts for the change in sign of the phases recorded in the blue 

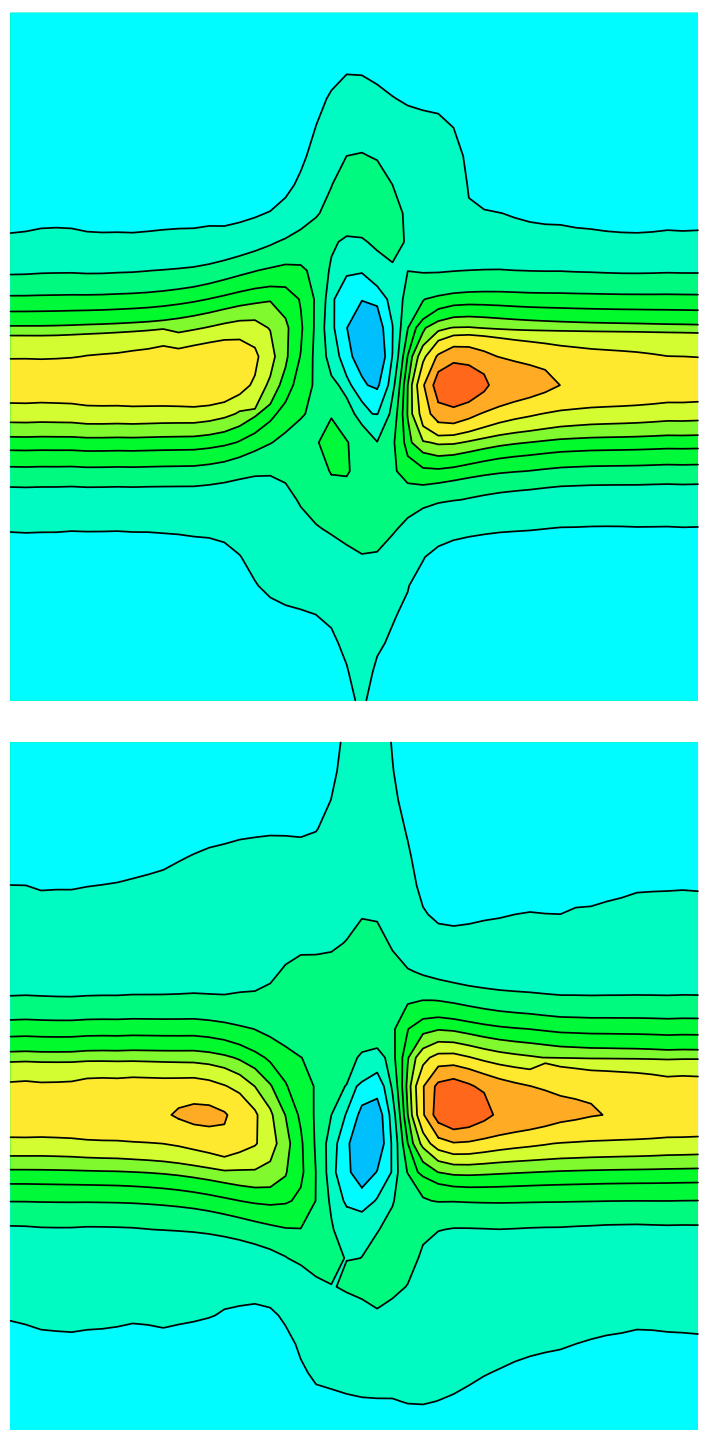

Fig. 11. Two top figures: cross-correlation functions obtained for symmetrical positions on the Sun, at $r= \pm 0.5 R_{\odot}$ (top: north; bottom: south). The horizontal axis corresponds to the spectral domain around the Fe I $557.6 \mathrm{~nm}$ line (bottom figure). The vertical axis corresponds to the displacements (total excursion of $\pm 3.4 \mathrm{arcsec}$ ). There is a wellmarked symmetry between the north and south curves, in particular if one follows the watershed into the blue deep hole corresponding to an anti-correlation peak near the line center. By continuity, the transition regions between correlation and anti-correlation peaks correspond to zones of lack of correlation between images.

and red wings of spectral lines. We show that this can be interpreted as the effect of unresolved granular velocity fields. We also present some correction procedures, based on simple assumptions about the structure of these unresolved fields. Finally we present the results obtained for the corrected shifts both in a weak line and in the strong Fe I $557.6 \mathrm{~nm}$ line. For the weak line, the shifts are derived from the phase of the cross-spectrum (as in Fig. 2) and then corrected for the velocity effects, whereas for the strong line we use the shifts derived from the sequential cross-spectrum (as in Fig. 3). The successive shifts measured along the line profile are corrected for the velocity field effect and then integrated from the continuum to the line.

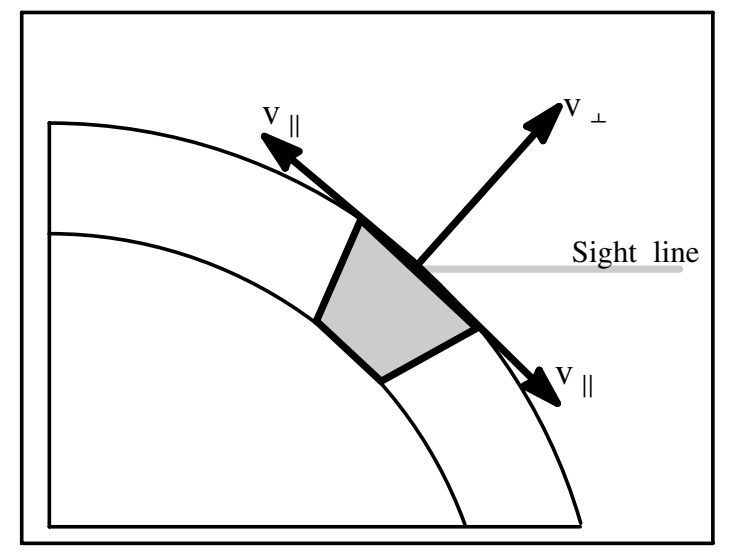

Fig. 12. Effect of the projected unresolved velocity fields in a granule. The horizontal components $v \|$ are antisymmetric with respect to the granule center. They have an opposite effect on the two granule edges. The vertical components $v \perp$ have the same positive contribution on the whole granule.

\section{Unresolved velocity fields effects and correction procedures}

\subsection{Effect of unresolved velocity fields: a simple model}

The change in sign of the phase of the cross-spectrum from the red to the blue wing of spectral lines cannot be due to a perspective effect since both wings are formed at higher altitudes than the continuum. As we explain now, opposite shifts in the two wings can be interpreted as the effects of uncorrected granular velocity fields.

The physical model is the following. A granule expands during its ascension from the bottom of the photosphere to the most external layers. This necessarily implies the existence of horizontal velocities, seen in projection by the observer, and of velocity gradients. We further assume that these horizontal velocities are not spatially resolved in the spectrograms and therefore not corrected by the pre-processing procedure of the data (correction of the Doppler displacements of the line). We recall that the highest spatial frequencies that we detect in our spectrograms are about $1.2 \mathrm{c} / \mathrm{s}$, which corresponds to spatial scales of $0.8 \mathrm{arcsec}$. This is not enough to fully resolve the velocity fields due to granular expansion. The velocity gradients are also not taken into account in our pre-processing. These unresolved velocities are responsible for the line broadening and asymmetry.

Let us consider a granule with a divergent and centered horizontal velocity field and depth-independent vertical velocity. Projected on the line of sight, horizontal velocity components move away from or come toward the observer, so the line is displaced either towards the red wavelengths or towards the blue ones, according to the granule edge we consider (see Fig. 12).

For the sake of clarity of presentation, we consider a very simplified model for the spectrogram $G(x, \lambda)$ corresponding to a granule. We first assume that the granule has the same shape $g(x)$ in both the line and the continuum. This may appear inconsistent with the expanding granule, but while expansion is a main feature of velocities, it may be considered as a secondorder term for the brightness distribution. This approximation can be easily withdrawn in a numerical simulation.

For each point of the granule, we assume an identical line profile $L(\lambda)$, shifted in wavelength by the local velocities. 
For a granule at the heliocentric angle $\theta$, the projected velocity to the observer can be written as

$v_{\theta}(x)=v_{\perp}(x) \cos (\theta)+v_{\|}(x) \sin (\theta)$,

where $v_{\perp}(x)$ and $v_{\|}(x)$ correspond to the vertical and to the horizontal velocities, as shown in Fig. 12.

To account for the line shape and asymmetry, we determine the line profile $L(\lambda)$ from an average of the observed $557.4 \mathrm{~nm}$ $\mathrm{Cr}$ I line profiles. We choose this rather weak line because as it is formed on a small thickness (from an LTE transfer code, we determine that for this line, $h=15 \mathrm{~km}$ ), we can assume that neither the shape of the simulated granule nor velocity moduli $v_{\|}$ and $v_{\perp}$ change a lot with depth. The order of magnitude of horizontal velocities is taken to be about $1 \mathrm{~km} \mathrm{~s}^{-1}$. For a weak line we may write the brightness distribution of the granule observed at the wavelength $\lambda$ as the product of the continuum brightness by a wavelength-dependent attenuation coefficient due to line absorption:

$G_{\theta}(x, \lambda) \sim g(x) L\left[\lambda\left(1-v_{\theta}(x) / c\right)\right]$.

The two components of $v_{\theta}(x)$ show a different behavior. In a simple model, we can assume that the pre-processing has compensated for the vertical velocities and that the displacement of the line on the scale of a granule is entirely due to the horizontal expansion. Residual uncorrected vertical velocities could be easily introduced in our model, but since we can suppose their distribution in space is symmetrical compared to the maximum of the granule brightness, they can only produce a difference in the shape of the granule and not cause the deterministic opposition of the shift we observe.

A possible representation for $G_{\theta}(x, \lambda)$ is drawn in Fig. 13, there we assume a simple Gaussian shape for the granular intensity $g(x)$. Moreover, the horizontal velocity $v_{\|}(x)$ varies linearly as $v_{\|}(x) \sim v_{0}\left(x-x_{0}\right)$, for a granule centered on the position $x_{0}$. For a granule centered on $x_{0}=0$, we have:

$$
\begin{aligned}
G_{\theta}(x, \lambda) \simeq & \exp \left(-\frac{x^{2}}{\sigma_{x}^{2}}\right) \\
& \times\left(1-b \exp \left(-\frac{\left(\lambda-\lambda_{0}-x \sin (\theta) \lambda_{0} v_{0} / c\right)^{2}}{\sigma_{\lambda}^{2}}\right)\right),
\end{aligned}
$$

where $b$ gives the strength of the line. As a consequence, not only is the amplitude of the granule brightness reduced in the line, but its shape is also modified because of the $x$-dependence of the exponential term. For the model written in the above equation, the shape modification consists in the subtraction of a Gaussian term, and the granule photocenter is shifted in opposite directions in the blue or red wings (see top panel of Fig. 13). In the wings of the line, the granules seem to be displaced to the north edge or the south edge on the slit, in comparison with the granules that are observed in the continuum.

We realize that this model gives a crude representation of the granulation. It is well-known that the inhomogeneities of the photosphere between granules and inter-granules in temperature, density, and velocity pattern, must be taken into account in the modeling of spectral lines. Synthetic spectrograms should be obtained from 3D models of the solar photosphere.

However, our simple modeling does provide us with a simulated spectrogram. We computed the cross-spectrum of the spectrogram in the continuum and in the line wing. The behaviors of the shifts in the blue and red wings are consistent with the observations, we can recover the right order of magnitude of the shifts for realistic values of the granular velocities. First, as seen (a) Shift of the granule photocenter

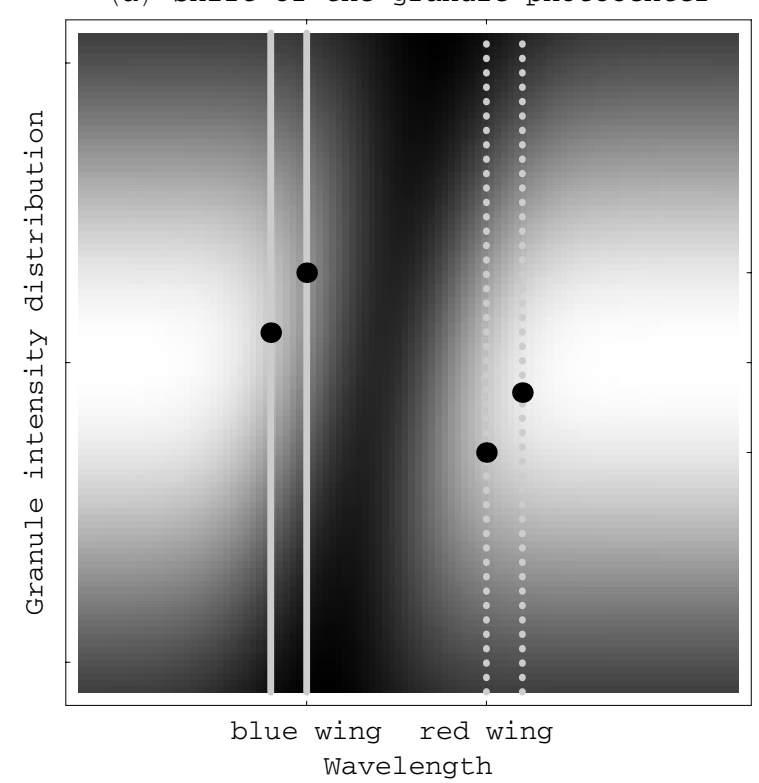

(b) Granule shape through the line

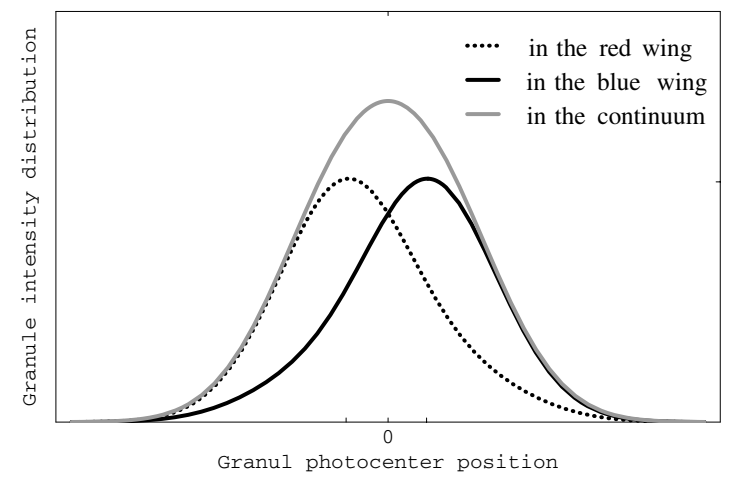

Fig. 13. Top: illustration of the spectrogram $G_{\theta}(x, \lambda)$ of an expanding granule; the slit of the spectroscope is vertical. Bottom: representation of the granule intensity distributions for the continuum and the two wavelengths represented by the vertical lines in the top figure. The observation is for the north hemisphere; the "red" granule photocenter is shifted towards South and the "blue" granule photocenter towards North.

in Fig. 14, we find opposite shifts for opposite positions on the Sun disk. Then, the shifts do have opposite signs in the blue and the red wings. The perspective effect and the effect from velocity fields add up. Indeed, the cross-spectrum phase is very sensitive to the disturbing effect of the unresolved velocity fields on the order of $1 \mathrm{~km} \mathrm{~s}^{-1}$. The perspective effect only appears as a secondorder term, which is at the origin of the slight asymmetry of the displacement with respect to line center.

\subsection{Correction procedures}

We now present simple correction procedures that are based on the assumption that the unresolved velocity field is a divergent horizontal flow centered on the center of the granule. The correction strategy is slightly different if we work with the shifts directly derived from the phase of the cross-spectrum or if we "reconstruct" them by integrating the elementary shifts obtained with the sequential cross-spectrum between two successive wavelengths. We recall that this second method is relevant for strong lines where contrast inversion is detected in the line core. 


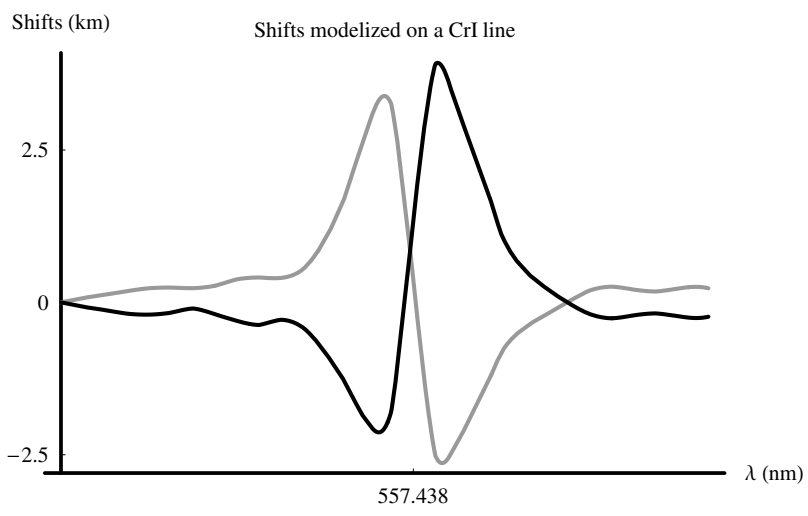

Fig. 14. Black (resp. gray) line: shifts calculated on South (resp. North) position. Shifts obtained taking in account both a perspective effect and an effect of horizontal velocities. The main effect is caused by the velocity fields. The perspective effect induces the small asymmetry between the shifts in the two wings.

\subsubsection{Weak lines}

The simple modeling presented above shows that our observations may be reproduced by the combined effect of two different mechanisms. The divergent granular velocity fields produce antisymmetrical shifts at symmetrical wavelengths with respect to line center, whereas the perspective effect is symmetrical. In order to eliminate the displacements due to velocities, we try to simply add the shifts obtained in both wings at the same depression level $\delta(\lambda)=\left(I_{\mathrm{C}}-I_{\mathrm{W}}(\lambda)\right) / I_{\mathrm{C}}$, where $I_{\mathrm{C}}$ is the intensity in the continuum and $I_{\mathrm{W}}(\lambda)$ the intensity in the line. We expect to observe the perspective effect as an increasing shift going from zero in the continuum (line depression equal to 0 ), to a maximum at line center (maximum line depression, depending on the line strength).

We applied this correction to the shifts measured for the weak Fe I line at $557.7 \mathrm{~nm}$. The results are shown in Fig. 15 and in Table 1. We see that the corrected shifts do not have the expected monotonous variation as a function of the line depression level. For depression levels close to the continuum $(0<\delta(\lambda)<0.07)$, the sign of the shift is the opposite of what we expect from the perspective effect. Several problems may contribute to spurious determination of the shifts in that domain. The continuum level is not precisely defined, and we suspect the presence of small blends close to the line; therefore, it is difficult to accurately determine the line level close to the continuum. Furthermore because of these blends, the "continuum" on the red side of the line may be formed at a slightly different altitude than the "continuum" on the blue side. This may be the reason the shift does not go exactly to zero when $\delta$ goes to zero (in Fig. 15, the shift is on the order of $-0.004 \operatorname{arcsec}$ at $\delta=0$ ). The line depression level is not accurately determined close to the continuum; as a consequence the correction procedure we implemented may give spurious results.

In the line core $(\delta(\lambda)>0.07)$, we observe a monotonous increase in the shift (in absolute value), with a roughly symmetrical behavior for north and south positions on the solar disk, as expected from a perspective effect. Notice that the northsouth symmetry of the results is quite well recovered if we measure the shifts with respect to the "effective" continuum level at -0.004 arcsec.

Assuming that we indeed measure the perspective effect at line center, we can derive the line formation depth, as compared to the continuum formation depth, at different positions on the

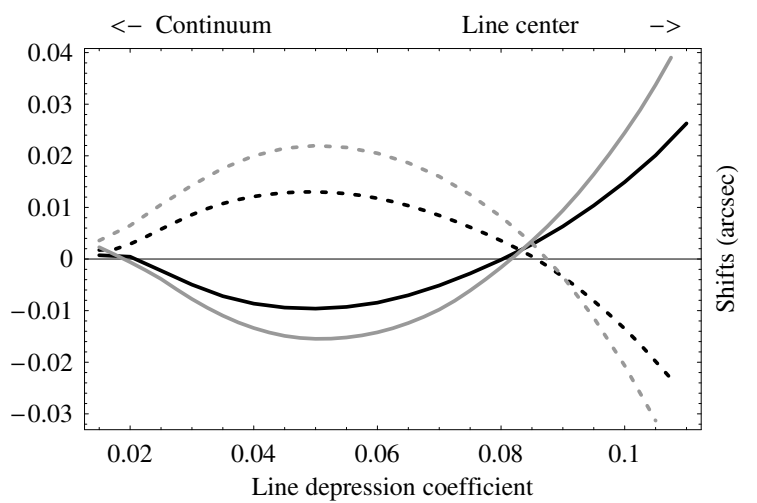

Fig. 15. Shifts corrected from the horizontal velocity effect, calculated on the Fe I $557.7 \mathrm{~nm}$ line. Dark line (resp. dark-dotted line): $r=0.5 R_{\odot}$ (resp. $r=0.75 R_{\odot}$ ) Gray line (resp. gray-dotted line): $r=-0.5 R_{\odot}$ (resp. $r=-0.75 R_{\odot}$ ) As expected, the closer to the solar edge we observe, the bigger the shifts are.

Table 1. Depth formation of the $557.7 \mathrm{~nm}$ iron line.

\begin{tabular}{|c|c|c|c|c|}
\hline \multicolumn{5}{|c|}{$557.7 \mathrm{~nm}$ iron line depth formation } \\
\hline$r / R_{\odot}$ & 0.5 & -0.5 & 0.75 & -0.75 \\
\hline$\Delta h(\mathrm{~km})$ & 24.4 & 29.6 & 28.1 & 34.2 \\
\hline mean $\Delta h(\mathrm{~km})$ & \multicolumn{2}{|c|}{27.0} & \multicolumn{2}{|c|}{31.2} \\
\hline theoretical ${ }^{a} \Delta h(\mathrm{~km})$ & \multicolumn{2}{|c|}{13.0} & \multicolumn{2}{|c|}{15.0} \\
\hline \multicolumn{5}{|c|}{$557.7 \mathrm{~nm}$ iron line depth formation ${ }^{b}$} \\
\hline$r / R_{\odot}$ & 0.5 & -0.5 & 0.75 & -0.75 \\
\hline$\Delta h(\mathrm{~km})$ & 27.7 & 26.3 & 31.9 & 30.6 \\
\hline mean $\Delta h(\mathrm{~km})$ & \multicolumn{2}{|c|}{26.5} & \multicolumn{2}{|c|}{31.3} \\
\hline
\end{tabular}

${ }^{a}$ Calculated with a $1 \mathrm{D}$ transfer code.

${ }^{b}$ Corrected from the continuum residual shift.

solar disk. The results are summarized in Table 1 and compared to the formation depth obtained by an LTE calculation of the line in a standard 1D quiet-sun model. Let us first notice that the results, once corrected for the continuum offset, are quite consistent: we obtain the same values for symmetrical north and south positions. When comparing to the predictions of $1 \mathrm{D}$ models, we find that the observed formation depth is larger by a factor of two. Such a discrepancy is not surprising; since 1D solar models are derived from low spatial-resolution observations, they are designed to reproduce spatial averages of the physical parameters, whereas we are observing small structures on the granular scale, where the physical parameters may have quite large fluctuations with respect to the spatial averages. High angular-resolution investigations of the solar photosphere, such as the one presented here, should be compared to more realistic 3D line calculations using 3D models of the solar granulation.

\subsubsection{Strong lines}

Let us now describe the procedure we have implemented to derive corrected shifts from sequential cross-spectra. The elementary shifts between two successive wavelengths along the line profile are due both to the perspective effect and to the effect of unresolved velocity fields. Let us examine the situation sketched in Fig. 13, which illustrates the photocenter displacements due to divergent flows. If we consider two successive wavelengths in the blue wing of the line, the velocity-induced relative displacement of the photocenter at the larger wavelength is positive, as is the displacement due to the perspective effect (we go up in the photosphere in the north hemisphere). If we now consider 


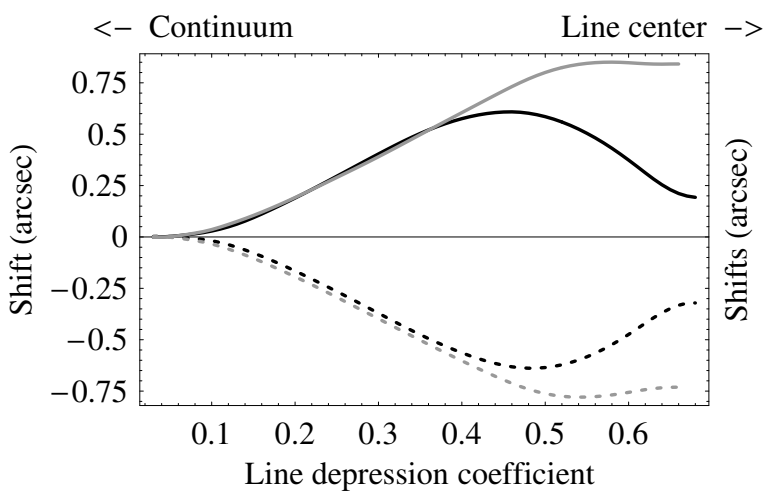

Fig. 16. Results for the Fe I $557.6 \mathrm{~nm}$ line. Plain lines: corrected shifts on the North hemisphere (dark for $r=0.5 R_{\odot}$ and gray for $r=0.75 R_{\odot}$ ). Dotted lines: corrected shifts on the South hemisphere (dark for $r=$ $-0.5 R_{\odot}$ and gray for $r=-0.75 R_{\odot}$ ).

Table 2. Depth formation of the $557.6 \mathrm{~nm}$ iron line.

\begin{tabular}{lcccc}
\hline \hline \multicolumn{5}{c}{$557.6 \mathrm{~nm}$ iron line depth formation } \\
\hline$r / R_{\odot}$ & 0.5 & -0.5 & 0.75 & -0.75 \\
$\Delta h(\mathrm{~km})$ & 441.5 & 616.1 & 992.8 & 846.4 \\
mean $\Delta h(\mathrm{~km})$ & \multicolumn{5}{c}{528.8} & 919.6 \\
theoretical ${ }^{a} \Delta h(\mathrm{~km})$ & \multicolumn{3}{c}{302} & \multicolumn{2}{c}{305} \\
\hline
\end{tabular}

${ }^{c}$ Calculated with the sequential cross-spectrum technique.

the two symmetrical wavelengths in the red wing, the velocityinduced relative displacement of the photocenter at the larger wavelength is still positive, as in the blue wing, whereas the perspective effect gives a negative displacement (we go down in the photosphere). We thus find that, as far as the sequential crossspectrum is concerned, the velocity-induced elementary shifts are symmetrical with respect to line center, whereas the perspective effect is anti-symmetrical. In order to correct the elementary shifts from the effect of velocities, we may simply subtract the shifts obtained for two symmetrical sets of wavelengths. Then we obtain the shift between the continuum and any wavelength in the line by integrating the corrected elementary shifts.

Figure 16 shows the corrected shifts as a function of the line depression level in the strong Fe I line at $557.6 \mathrm{~nm}$. The signs of the corrected shifts are consistent with what we expect for a perspective effect. We nevertheless observe a non monotonous behavior that is certainly due to residual velocity effects which are not well-corrected by our simple procedure. Table 2 gives the line formation depth at line center derived from the corrected shifts shown in Fig. 16. We notice that the results are quite different for two symmetric positions with respect to the solar disk center. They are also significantly larger than the line formation depth derived from the LTE calculation of the line formation, in a 1D quiet solar model.

Both these discrepancies and the non-monotonous variation of the corrected shifts show that our treatment of the small-scale velocity fields is not precise enough to extract the shift due to a perspective effect. In particular we do not take into account velocity gradients that give rise to the asymmetry of the line profile. A more refined treatment of the velocity effects should be implemented, in particular for strong lines formed over large depths in the photosphere. This requires a better image quality than what we could get during the observing run that we present here.

\section{Conclusion}

This paper reports on the first use of DSI for solar observations. It confirms the feasibility of such a study and its broad interest. First, the DSI technique is not diffraction-limited. It allows very small displacements on the Sun (on the order of 0.01 arcsec) to be measured directly from the phase term of the cross-spectrum calculated between 1D intensity variations observed in the continuum and in spectral lines. The phase term is, afterall, not affected by the atmospheric fluctuations.

In this paper, our aim was to explore this new tool for the $3 \mathrm{D}$ investigation of the photospheric solar structures. We encountered several kinds of difficulties. First, we needed to correct the spectrograms from Doppler displacements of the lines due to granular velocities. This correction is very sensitive to the image quality. The uncorrected velocity fields induce uncorrected shifts that are detectable with the DSI technique. As a consequence, instead of measuring shifts caused only by a perspective effect, which would go from 0 in the continuum to a maximum at line center, we systematically observed opposite shifts in the two wings.

We tried several correction procedures to eliminate the small-scale velocity effect. They rely on the rather crude assumption that the velocity field on a small scale is a divergent flow centered on the center of the granules. More refined corrections should be implemented, in order to achieve more accurate measurements of the perspective effect.

For strong lines we detect a contrast inversion between the lower photosphere where the continuum is formed and the upper photosphere where the line core is formed. The signature of this contrast inversion clearly appears in the phase of the continuum/line core cross-spectrum, as a jump of $\pm \pi$ of the phase. It is detected for spatial frequencies larger than 0.4 cycle/s, i.e. spatial scales smaller than 2.5 arcsec. We also checked that the crosscorrelation of the spectrograms shows an anti-correlation peak in the line core. This phenomenon is present in the $3 \mathrm{D}$ numerical simulations of the solar photospheric convection. In such conditions, the shift of the structures in the continuum and in the line core cannot be measured on the phase of the cross-spectrum. We implemented a different procedure based on the calculation of the sequential cross-spectrum between two nearby wavelengths on the line profile.

The DSI technique could also be applied to 2D images obtained at different wavelengths along a spectral line. However we stress that it requires that the images in the continuum and in the absorption line are taken precisely at the same time and that they can be rigorously superimposed. Finally, as the accuracy of the velocity field corrections depends crucially on the image quality, this technique would greatly benefit from new observations, obtained with a shorter integration time and in better seeing conditions.

Acknowledgements. The experiment realized at THEMIS in 2002 profited from a first experiment done at the Vacuum Tower Telescope by one of us (CA) with Rémi Soummer and Oskar Von der Lühe, which rose very interesting discussions that influenced several aspects of the observations and data processing used here. C. Grec wishes to thank the THEMIS direction for supporting her observing run in 2005 .

\section{Appendix A: Phase behavior at high and low spatial frequencies}

In this appendix, we propose a possible explanation of the particular behavior of the cross-spectrum phase in high and low spatial frequencies. 


\section{A.1. High frequencies}

The phase term returns to zero in the high-frequencies for all cross-spectra. This effect is indeed surprising, since we expected to see a random noise there, because there is no more signal in that region. The random value of the phase is effectively observed in stellar measurements (Aime et al. 1984). We propose explaining this effect as the result of a residual uncorrected flatfield term. The micro-defects existing along the spectrograph slit produce extremely small and systematic structures in the images (on the order of one pixel large). It induces a systematic correlation in the cross-spectrum. This term is dominant when the solar signal disappears.

Let us denote the micro-defects $\alpha(x)$ and the transmission function along the slit $s(x)=1+\alpha(x)$. The solar signal is denoted by $1+g(x)$ in the continuum and by $1+g(x-\varepsilon)$ in the line. The resulting signal in the continuum $\left(I_{\mathrm{C}}(x)\right)$ and in the line $\left(I_{\mathrm{W}}(x)\right)$ is

$I_{\mathrm{C}}(x) \simeq(1+\alpha(x)+g(x))$,

$I_{\mathrm{W}}(x) \simeq(1+\alpha(x)+g(x-\varepsilon))$,

where we have neglected the second-order terms $\alpha(x) g(x)$ and $\alpha(x) g(x-\varepsilon)$. After the subtraction of the continuum and the Fourier transform, the main terms are

$\widehat{I}_{\mathrm{C}}(u) \simeq \widehat{\alpha}(u)+\widehat{g}(u)$,

$\widehat{I}_{\mathrm{W}}(u) \simeq \widehat{\alpha}(u)+\widehat{g}(u) \mathrm{e}^{2 \mathrm{i} \pi u \varepsilon}$,

so we can write the cross-spectrum as

$\widehat{Q}_{\mathrm{CW}}(u)=\left\langle\widehat{I}_{\mathrm{C}}(u) \widehat{I}_{\mathrm{W}}^{*}(u)\right\rangle$,

$\widehat{Q}_{\mathrm{CW}}(u) \simeq\left\langle|\widehat{g}(u)|^{2}\right\rangle \mathrm{e}^{-2 \mathrm{i} \pi u \varepsilon}+\left\langle|\widehat{\alpha}(u)|^{2}\right\rangle$.

We have suppressed the zero-averaged cross-terms in Eq. (A.5). The phase term $\mathrm{e}^{-2 \mathrm{i} \pi u \varepsilon}$ disappears at high frequencies since $\left\langle\left.\widehat{g}(u)\right|^{2}\right\rangle$ contributes weakly, so the only contribution in high frequencies is $\left\langle|\widehat{\alpha}(u)|^{2}\right\rangle$, which has a null phase term.

\section{A.2. Low frequencies}

We observe a change of slope of the phase for $|u| \leq 0.2$ cycle/s. This can be explained by the low quality of our images. The domain of isoplanatism is much smaller than the length of the slit $\left(70^{\prime \prime}\right)$, which results in the convolution of the cross-spectrum in Fourier space. We verified by a simple simulation that this convolution produces a break in the phase at low frequencies. The phase remains linear, but with a different (smaller) slope. This effect would be less in better seeing conditions.

\section{References}

Aime, C. 1974, J. Opt. Soc. Am., 64, 1129

Aime, C. 1978, A\&A, 67, 1

Aime, C., Martin, F., Petrov, R., Ricort, G., \& Kadiri, S. 1984, A\&A, 134, 354 Aime, C., Petrov, R., Martin, F., Ricort, G., \& Borgnino, J. 1986, Opt. Eng., 25, 716

Asplund, M., Nordlund, Å., Trampedach, R., \& Stein, R. F. 2000, A\&A, 359, 743 Beckers, J. M., \& Hege, E. K. 1982, in IAU Coll. 67: Instrumentation for Astronomy with Large Optical Telescopes, ed. C. M. Humphries, ASSL, 92, 199

Eibe, M. T., Mein, P., Roudier, T., \& Faurobert, M. 2001, A\&A, 371, 1128

Harvey, J. W., \& Breckinridge, J. B. 1973, ApJ, 182, L137

Janssen, K., \& Cauzzi, G. 2006, A\&A, 450, 365

Knox, \& Thompson 1974, A\&A, 182, L137

Krieg, J., Kneer, F., Koschinsky, M., \& Ritter, C. 2000, A\&A, 360, 1157

Labeyrie, A. 1970, A\&A, 6, 85

Nordlund, A. 1985, Sol. Phys., 100, 209

Petrov, R., Roddier, F., \& Aime, C. 1986, Opt. Soc. Am. J. A, 3, 634

Prieur, J.-L., Avila, R., Daigne, G., \& Vernin, J. 2004, PASP, 116, 778

Ricort, G., \& Aime, C. 1979, A\&A, 76, 324

Von der Lühe, O., \& Pehlemann 1988, A\&A, 612, L85

Weigelt 1977, A\&A, 182, L137

Wilken, V., de Boer, C. R., Denker, C., \& Kneer, F. 1997, A\&A, 325, 819 